\title{
Avaliação dos usuários sobre o funcionamento de uma clínica odontológica universitária
}

\author{
Anne Gabryelle Marques de Oliveira Lima*; Suianny Fauth*; Edla Helena Salles de Brito**; \\ Paulo Leonardo Ponte Marques***

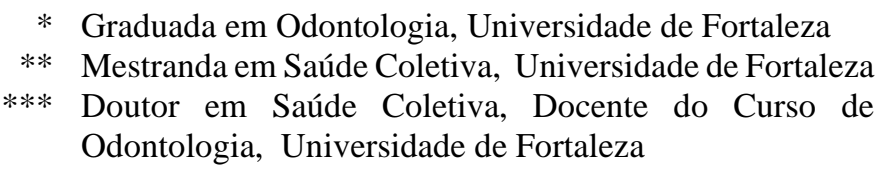

Recebido: 13/07/2020. Aprovado: 28/06/2021.

\begin{abstract}
RESUMO
Este estudo teve por objetivo conhecer a percepção dos usuários sobre a qualidade dos serviços prestados na clínica odontológica de uma universidade privada. Trata-se de estudo transversal de abordagem quantitativa, que teve como cenário uma universidade do nordeste brasileiro. A coleta de dados foi realizada por meio de questionário com 35 perguntas objetivas relacionadas à qualidade do atendimento clínico, incluindo aspectos estruturais e organizacionais da instituição de ensino, aplicados na recepção das clínicas, no período de fevereiro a novembro de 2019. Dentre os 203 participantes, $196(96,6 \%)$ sentiram-se respeitados pelos profissionais; 195 (96,0\%) informaram que nunca tiveram o atendimento interrompido por falta de material ou problema com equipamentos; e $201(99,0 \%)$ recomendariam o atendimento clínico a amigos e familiares. Sobre o conforto durante atendimento, $183(90,1 \%)$ sentiram-se satisfeitos e $177(87,2 \%)$ avaliaram as instalações da clínica como muito boas. A percepção dos usuários da clínica odontológica universitária foi considerada muito boa quanto ao acolhimento; positiva quanto ao vínculo e satisfação no atendimento; e com quantidade de insumos e equipamentos suficientes para prestação de serviço com qualidade.

Descritores: Ensino Odontológico. Assistência Odontológica. Controle de Qualidade. Satisfação do Paciente.
\end{abstract}

\section{INTRODUÇÃO}

Visto que a oferta de serviços clínicos odontológicos para população não garante que esses sejam realizados com qualidade, faz-se necessário utilizar parâmetros com vistas a avaliar e aprimorar a assistência à saúde. O processo avaliativo é concretizado por meio de ações, que verificam o cumprimento de padrões préestabelecidos pelo governo ou instituições, buscando a melhoria no acesso e na qualidade dos serviços prestados em saúde ${ }^{1}$.

Do ponto de vista acadêmico, as universidades se concentram em realizar avaliações coletando opiniões de docentes e discentes, deixando eventualmente em segundo plano a opinião dos pacientes, que exercem um papel de extrema importância nas clínicas-escola. Esses pacientes não devem ser entendidos como um mero instrumento de aprendizado, devem ser tratados com respeito e receber o tratamento 
indicado e sentirem-se satisfeitos em todos os aspectos envolvidos no seu cuidado ${ }^{2}$.

As pesquisas em relação à satisfação dos usuários propiciam melhora no cotidiano dos serviços prestados, pois a visão desses constitui um importante instrumento de diagnóstico da realidade, com a possibilidade de intervenção para a melhoria na assistência de qualidade ${ }^{3}$. A percepção dos usuários a respeito do atendimento odontológico prestado pelos universitários e serviços em geral permite a melhor compreensão dos fatores relacionados à assistência de qualidade. Além de receber um retorno sobre os serviços ofertados e eventual melhora no desempenho da função dos prestadores de serviços de saúde, o desenvolvimento da relação profissional-paciente é de fundamental importância para estabelecer entendimento entre as partes envolvidas, já que quanto mais o profissional entende o que o paciente procura, mais favorável será o atendimento ${ }^{4,5}$.

Estudos evidenciam que a busca aos serviços e satisfação dos usuários não está intimamente ligada aos aspectos técnicos e científicos propriamente ditos, e sim com a maneira como são tratados e com a resolutividade dos problemas bucais $^{6}$. É indiscutível o papel norteador das avaliações dos serviços de saúde em relação ao desempenho dos prestadores de serviço. O processo avaliativo deve servir como um instrumento de transformação na busca pelo avanço na qualidade do atendimento ${ }^{7}$.

A avaliação é uma forma de contribuição da construção e do aperfeiçoamento, devendo ser permanente, sistemática, dinâmica e articulada às ações implementadas e buscar a identificação das dificuldades, a orientação de estratégias e a organização dos serviços de saúde. Constitui-se numa ferramenta indispensável para a qualificação das ações e dos cuidados das pessoas, da família e da comunidade como um todo ${ }^{8}$.

Assim, este estudo teve por objetivo conhecer a percepção dos usuários sobre a qualidade dos serviços prestados na clínica odontológica de uma universidade privada.

\section{MÉTODOS}

Trata-se de estudo transversal quantitativo, que teve como cenário a clínica odontológica de uma universidade privada localizada na cidade de Fortaleza/CE; previamente aprovado pelo Comitê de Ética em Pesquisa institucional (parecer 3.192.914, CAAE 7604918.3.0000.5052). Todos os participantes da pesquisa assinaram Termo de Consentimento Livre Esclarecido.

Criada em 1973, a Universidade de Fortaleza conta com 25 mil alunos e cerca de 1.200 professores de diversos cursos na área de graduação e pós-graduação. Foi classificada como a melhor instituição de ensino superior particular do Norte e Nordeste, segundo o Ranking Universitário Folha 2019, e está entre as melhores universidades do mundo com até 50 anos, segundo o Times Higher Education Young University Ranking 2020. Entre os 40 cursos de graduação se encontra o curso de Odontologia. Implantado em 1995, conta com 100 consultórios odontológicos, central de esterilização com funcionamento 24 horas, além de laboratórios, setor de radiologia e salas de aula.

A coleta de dados foi realizada por meio de um questionário que tomou como referência as perguntas utilizadas no formulário de avaliação adotado pelo Programa Nacional de Melhoria da Qualidade (PMAQ), do Ministério da Saúde. Os dados foram coletados por uma única pesquisadora treinada para aplicação do questionário, com o propósito de evitar interferências nas respostas. Os participantes foram convidados para a pesquisa nas dependências e recepção da clínica em dias úteis, nos períodos da manhã, tarde e noite.

O cálculo amostral a partir do quantitativo de pacientes estimados (412) para atendimento na clínica odontológica no primeiro semestre de 2019. Considerando um nível de significância de $95 \%$ e 
erro amostral de 0,05 resultou em 203 participantes. Foram incluídos, aleatoriamente, pessoas maiores de 18 anos que já tivessem recebido pelo menos um atendimento na clínica.

A coleta foi realizada no ano de 2019, entre os meses de fevereiro e novembro. Os dados foram consolidados e organizados em planilha eletrônica. A análise foi realizada por meio de estatística descritiva utilizando-se frequência, medida de tendência central (média) e medida de dispersão (desvio padrão).

\section{RESULTADOS}

A tabela 1 contém os dados do perfil dos participantes, na maioria do sexo feminino $(\mathrm{n}=143 ; 70,4 \%)$; idade entre 18 e 82 anos $(42,8 \pm$ 15,4 anos), solteiros (n =91; 44,8\%); autodeclarados brancos (n $=79 ; 38,9 \%)$; residentes no município de Fortaleza ( $\mathrm{n}=189$; 93,1\%); com pelo menos o nível médio de escolaridade completo $(n=110 ; 54,2 \%)$; trabalho remunerado ( $\mathrm{n}=114 ; 56,2 \%)$; e renda familiar entre 1 e 2 salários mínimos $(n=102 ; 50,2 \%)$.

Tabela 1. Perfil dos participantes.

\begin{tabular}{llcc}
\hline \multicolumn{1}{c}{ Variáveis } & & n & \% \\
\hline Sexo & Masculino & 60 & 29,6 \\
Estado civil & Feminino & 143 & 70,4 \\
& Solteiro & 91 & 44,8 \\
& Casado/União estável & 89 & 43,9 \\
& Separado/Divorciado & 15 & 7,4 \\
Mora no munícipio & Viúvo & 8 & 3,9 \\
& Sim & 189 & 93,1 \\
Grau de instrução & Não & 14 & 6,9 \\
& Não alfabetizado & 4 & 2,0 \\
& Alfabetizado & 12 & 5,9 \\
& Ensino fundamental incompleto & 34 & 16,7 \\
& Ensino fundamental completo & 33 & 21,2 \\
& Ensino médio completo & 84 & 41,3 \\
Trabalho remunerado & Superior completo & 16 & 7,9 \\
\multirow{2}{*}{ Renda } & Pós-graduação & 10 & 5,0 \\
& Sim & 114 & 56,2 \\
& Não & 89 & 43,8 \\
& Sem renda & 13 & 6,4 \\
& Menos de 1 salário & 50 & 24,6 \\
& De 1 a 2 salários & 102 & 50,2 \\
& Acima de 2 salários & 38 & 18,8 \\
\hline
\end{tabular}

Na tabela 2 observam-se os resultados do questionário quanto ao acolhimento e ao atendimento dos participantes. A maioria avaliou $o$ atendimento como muito bom $(n=176 ; 86,7 \%)$; afirmou ser sempre respeitada pelos profissionais $\quad(n=196 ; 96,6 \%)$ sentiu-se confortável durante o atendimento $(\mathrm{n}=183$;
90,1\%); recebeu orientação dos estudantes e professores $(\mathrm{n}=187 ; 92,1 \%)$ e conseguiu resolver seu problema odontológico $(n=134$; $66,0 \%)$.

Na tabela 3 estão apresentados os dados sobre vínculo e responsabilização. A maioria dos participantes informou que houve esclareci- 
mentos sobre seu tratamento $(n=200 ; 98,50 \%)$; foi tratado pelo nome $(\mathrm{n}=193 ; 95,1 \%)$; tinha facilidade para tirar dúvidas após as consultas ( $\mathrm{n}=107 ; 52,7 \%)$; nunca interrompeu o tratamento $(\mathrm{n}=115 ; 56,7 \%)$; não precisou ser encaminhado para outra clínica/especialidade $(\mathrm{n}=102 ; 50,2 \%)$; e que o cuidado recebido pelo estudante havia sido muito bom ( $\mathrm{n}=184 ; 90,6 \%)$. O atendimento recebido na recepção foi considerado muito bom por 168 participantes $(82,7 \%)$.

Tabela 2. Avaliação dos participantes quanto ao acolhimento e atendimento

\begin{tabular}{llcc}
\hline \multicolumn{1}{c}{ Variáveis } & & n & \% \\
\hline & Muito bom & 176 & 86,7 \\
Acolhimento na clínica odontológica & Bom & 20 & 9,9 \\
& Razoável & 5 & 2,5 \\
& Ruim & 2 & 0,9 \\
É respeitado pelos profissionais & Sempre & 196 & 96,6 \\
& Às vezes & 6 & 2,9 \\
Conforto durante atendimento no consultório & Não & 1 & 0,5 \\
& Muito bom & 183 & 90,1 \\
& Bom & 18 & 8,9 \\
É orientado pelos profissionais sobre os cuidados na recuperação & Razoável & 2 & 1,0 \\
& Sempre & 187 & 92,1 \\
& Às vezes & 15 & 7,4 \\
Concluiu algum tratamento na clínica & Nunca & 1 & 0,5 \\
& Sim & 93 & 45,8 \\
Considera seu problema odontológico & Não & 110 & 54,2 \\
& Resolvido & 134 & 66,0 \\
& Não resolvido & 69 & 34,0 \\
\hline
\end{tabular}

Tabela 3. Avaliação dos participantes quanto ao vínculo e responsabilização

\begin{tabular}{|c|c|c|c|}
\hline Variáveis & & $\mathbf{n}$ & $\%$ \\
\hline \multirow[t]{2}{*}{ Houve esclarecimentos sobre seu tratamento } & Sim & 200 & 98,5 \\
\hline & Não & 3 & 1,5 \\
\hline \multirow{2}{*}{ Os estudantes lhe chamam pelo nome } & Sim & 193 & 95,1 \\
\hline & Não & 10 & 4,9 \\
\hline \multirow{3}{*}{ Lembram do que aconteceu nas últimas consultas } & Sim & 192 & 94,6 \\
\hline & Não & 5 & 2,5 \\
\hline & Não sabe & 6 & 2,9 \\
\hline \multirow{4}{*}{$\begin{array}{l}\text { Quando precisa tirar dúvidas após as consultas tem } \\
\text { facilidade }\end{array}$} & Sempre & 107 & 52,7 \\
\hline & Às vezes & 20 & 9,9 \\
\hline & Não & 4 & 2,0 \\
\hline & Não precisou & 72 & 35,4 \\
\hline \multirow{4}{*}{$\begin{array}{l}\text { Quando interrompe o tratamento é procurado para } \\
\text { retomá-lo }\end{array}$} & Sim, pelos funcionários & 44 & 21,7 \\
\hline & Sim, pelos estudantes & 35 & 17,2 \\
\hline & Não & 9 & 4,4 \\
\hline & Nunca interrompeu & 115 & 56,7 \\
\hline \multirow[t]{3}{*}{ Satisfação com o cuidado recebido pelo estudantes } & Muito bom & 184 & 90,6 \\
\hline & Bom & 19 & 9,4 \\
\hline & Regular/Ruim & - & - \\
\hline \multirow[t]{3}{*}{ Satisfação com o atendimento recebido na recepção } & Muito bom & 168 & 82,7 \\
\hline & Bom & 29 & 14,3 \\
\hline & Regular/Ruim & 06 & 3,0 \\
\hline
\end{tabular}


Na tabela 4 estão apresentados os resultados quanto a aspectos da estrutura da clínica odontológica. A maioria dos participantes respondeu que tinha cadeiras suficientes na recepção ( $n=190 ; 93,6 \%)$; a clínica estava em boas condições de limpeza $(n=200 ; 98,5 \%) ; \mathrm{e}$ as instalações da clínica eram muito boas $(n=177$; $87,2 \%)$. Quanto a ter o tratamento interrompido por falta de material, somente oito participantes (4\%) responderam que sim.

Quanto à possibilidade de realizar o tratamento odontológico em outro local, a maioria dos participantes $(n=186 ; 91,6 \%)$ assinalou que não buscaria. Para os que apontaram essa possibilidade, os motivos foram: local ser distante de casa $(\mathrm{n}=06 ; 35,3 \%)$, horário de atendimento não favorável $(n=4 ; 23,5 \%)$, por ter sido mal atendido $(\mathrm{n}=1 ; 6,0 \%)$, por outros motivos $(n=6 ; 35,2 \%)$.

No que diz respeito à credibilidade, a maioria $(\mathrm{n}=201 ; 99,0 \%)$ dos pacientes recomendaria a clínica para amigos ou familiares. $\mathrm{O}$ atendimento foi considerado muito bom pela maioria dos usuários $(\mathrm{n}=185 ; 91,1 \%)$.

Tabela 4. Avaliação dos participantes quanto a insumos, equipamentos, ambiente e credibilidade

\begin{tabular}{|c|c|c|c|}
\hline \multicolumn{2}{|l|}{ Variáveis } & $\mathbf{n}$ & $\%$ \\
\hline \multirow[t]{2}{*}{ Já teve o atendimento interrompido } & Sim & 8 & 4,0 \\
\hline & Não & 195 & 96,0 \\
\hline \multirow{2}{*}{ Tem cadeiras suficientes na recepção } & Sim & 190 & 93,6 \\
\hline & Não & 13 & 6,4 \\
\hline \multirow{2}{*}{ As instalações da clínica estão em boas condições de limpeza } & Sim & 200 & 98,5 \\
\hline & Não & 3 & 1,5 \\
\hline \multirow{3}{*}{ Como avalia as instalações da clínica } & Muito boas & 177 & 87,2 \\
\hline & Boas & 25 & 12,3 \\
\hline & Regulares/Ruins & 1 & 0,5 \\
\hline \multirow[t]{4}{*}{ Faria seu tratamento odontológico em outro local } & Sim & 17 & 8,4 \\
\hline & Não & 186 & 91,6 \\
\hline & Distante de casa & 6 & 35,3 \\
\hline & Horário de atendimento & 4 & 23,5 \\
\hline \multirow[t]{2}{*}{ Se sim, por quê? } & Mal atendido & 1 & 6,0 \\
\hline & Outros & 6 & 35,2 \\
\hline \multirow[t]{2}{*}{ Recomendaria essa clínica para amigo ou familiar } & Sim & 201 & 99,0 \\
\hline & Não & 2 & 1,0 \\
\hline \multirow{3}{*}{ Avaliação do atendimento } & Muito bom & 185 & 91,1 \\
\hline & Bom & 15 & 7,4 \\
\hline & Regular/Ruim & 3 & 1,5 \\
\hline
\end{tabular}

\section{DISCUSSÃO}

$\mathrm{O}$ agir profissional que reconhece o caráter humano e o social como intrínsecos ao ato de cuidar tem se tornado preponderante na relação que se estabelece entre o profissional e o usuário 9 . No presente estudo, sobre as características de acolhimento e atendimento aos participantes, muitos usuários consideraram muito bom o atendimento prestado na clínica odontológica e a maioria afirmou que se sente confortável durante o atendimento clínico.

Vale ressaltar que a clínica odontológica dessa universidade privada adota como protocolo, no início do atendimento, a entrega de 
um kit de proteção individual para os pacientes contendo itens descartáveis (gorro, babador e lenço para limpeza facial) e, adicionalmente, se fornece óculos de proteção individual. Estes cuidados adotados pela instituição seguem rigorosamente as regras de biossegurança ${ }^{10}$.

O acolhimento é constituído de forma coletiva, a partir da análise dos processos de trabalho e construção da relação de confiança, compromisso e vínculo entre as equipes/serviços, trabalhador/equipes e usuário com sua rede sócio afetiva $^{11}$. Na presente pesquisa, grande parcela dos entrevistados, 96,6\% dos usuários relataram sentir-se respeitados por todos os profissionais que tiveram contato, iniciando na recepção da clínica, em seguida com orientações recebidas pela assistente social, encaminhados para sala de triagem e, posteriormente, para o atendimento clínico por alunos da universidade.

O PMAQ destaca os processos de acolhimento e de vínculo nas práticas das equipes $^{12}$. Esses achados se tornaram presentes na investigação ao ficar evidenciado que os pacientes são tratados pelo nome e que, um elevado percentual de usuários (98,5\%), receberam esclarecimentos sobre seu tratamento durante o atendimento, inclusive, a maior parte dos pacientes $(56,7 \%)$ relatou nunca ter interrompido o tratamento.

Isso mostra um alto percentual de vínculo e o quanto os pacientes se sentem motivados e não se ausentam dos atendimentos agendados por alunos na clínica odontológica que, na maioria das vezes, são semanais. Um estudo feito no Rio Grande do Sul ressalta a importância do vínculo em relação à produção do cuidado nas práticas clínicas, caracterizadas por um acolhimento de escuta e diálogo com o usuário, que produz a pactuação de um plano terapêutico tornando o tratamento mais humanizado ${ }^{13}$.

Quanto à possibilidade de realizar o tratamento odontológico em outro local, grande parte $(91,6 \%)$ assinalou que não buscaria, pois se sentiam satisfeitos com os atendimentos recebidos pelos alunos e funcionários e, 99\% informaram que recomendariam a clínica odontológica universitária para amigos e ou familiares.

Esses resultados reforçam a confiança dos pacientes no serviço. Um estudo realizado na região Sul Fluminense do estado do Rio de Janeiro, ressaltou a importância do relacionamento entre profissional de saúde e paciente, pois, quanto mais o profissional entende a necessidade do usuário, mais favorável será $\mathrm{o}$ atendimento ${ }^{14}$.

Sabe-se que ter uma boa qualidade de insumos, equipamentos e ambiência é fundamental para que o paciente desfrute de um atendimento qualificado. Problemas nessa esfera organizativa estrutural comprometem a assistência e a plena utilização da capacidade instalada dos serviços existentes ${ }^{15,16}$. Sobre o aspecto estrutural, o estudo também comprovou a satisfação por parte dos usuários, pois $93,6 \%$ relataram contentamento com a quantidade de cadeiras disponíveis no local de espera pelo atendimento.

Ao serem indagados a respeito dos serviços de limpeza da clínica odontológica, grande parte dos usuários $(98,5 \%)$ afirmaram que a mesma estava em boas condições de limpeza, o que corrobora outros estudos, realizados em clínica odontológica universitária de outros estados ${ }^{17,18}$.

Este estudo teve como limitação a apresentação de resultados apenas descritivos e de ter sido realizado com participantes de diferenciados grupos etários não representativos, cujas respostas não podem ser generalizadas. Levando-se em consideração a importância de se conhecer a percepção dos usuários em relação à qualidade do serviço prestado, recomenda-se que a instituição sempre faça pesquisas aplicadas aos seus usuários objetivando mensurar a qualidade 
dos serviços ofertados, e se existe a necessidade de realizar melhorias.

\section{CONCLUSÃO}

A percepção dos usuários da clínica odontológica universitária foi considerada muito boa quanto ao acolhimento; positiva quanto ao vínculo e satisfação no atendimento; e com quantidade de insumos e equipamentos suficientes para prestação de serviço com qualidade.

\section{ASTRACT}

Users' assessment of the functioning of a university dental clinic

This study aimed to understand the users' perception of the quality of services provided in the dental clinic of a private university. This is a quantitative cross-sectional study carried out at a university in Northeastern Brazil. Data were collected using a questionnaire with 35 closedended questions on the quality of clinical care, including structural and organizational aspects of the education institution, applied at the reception of clinics from February to November 2019. Of the 203 participants, 196 (96.6\%) felt respected by the professionals; $195(96.0 \%)$ reported that they never had their service interrupted due to lack of material or problems with equipment; and $201(99.0 \%)$ said they would recommend clinical care to friends and family. Regarding comfort during care, 183 (90.1\%) felt satisfied and 177 $(87.2 \%)$ rated the clinic's facilities as very good. The users' perception of the university dental clinic was considered very good regarding user embracement; positive regarding the bond and satisfaction with the service; and there was a sufficient quantity of inputs and equipment to provide a quality service.

Descriptors: Education, Dental. Dental Care. Quality Control. Patient Satisfaction.

\section{REFERÊNCIAS}

1. Cerdeira LCR, Groisman S. Qualidade dos serviços de saúde bucal sob a perspectiva do usuário. Rev Bras Odontol. 2014;71(2):2037.
2. Toledo B, Campos AA, Leite RA. Análise da satisfação do paciente com o atendimento odontológico na Clínica de Odontologia da Universidade de Franca. Rev ABENO. 2010; 10(2):72-8.

3. Marinho NBP, Freitas RWJF, Lisboa QWSC, Alencar APG, Damasceno MMC. Avaliação da satisfação de usuários de um serviço especializado em diabetes mellitus. Rev Bras Enferm. 2018;71(1):599606.

4. Mota LQ, Farias DBLM, Santos TA. Humanização no atendimento odontológico: acolhimento da subjetividade dos pacientes atendidos por alunos de graduação em Odontologia. Arq Odontol. 2012;16(4):53744.

5. Ali DA. Patient satisfaction in dental healthcare centers. Eur J Dent. 2016; 10(3):309-14.

6. Guerra CT, Bertoz APM, Fajardo RS, Rezende MCRA. Reflexões sobre o conceito de atendimento humanizado em Odontologia. Arch Health Invest. 2014; 19(12):4777-86.

7. Galdino SV, Reis EMB, Santos CB, Soares FP, Lima FS, Caldas JG, Piedade MACR, Oliveira AS. Ferramentas de qualidade na gestão dos serviços de saúde: revisão integrativa de literatura. Gestão \& Saúde. 2016; 7(1):1023-57.

8. Pereira CG, Groisman, S. Histórico do monitoramento e avaliação da Estratégia de Saúde da Família no Brasil. Rev Bras Odontol. 2014; 71(2):208-10.

9. Warmling CM, Baldisserotto, J, Rocha, ET. Acolhimento e acesso de necessidades de saúde bucal e o agir profissional na Atenção Primária à Saúde. Interface - Comun Saúde Educ. 2019; 23: e180398. [Acesso em: 8 ago. 2020]. Disponível em: https://www.scielo.br/pdf/icse/v23/1807- 
5762-icse-23-e180398.pdf

10. Campos CAS, Guimarães Neto GSC, Pereira RS, Costa MOC, Santos WB, Rocha WGR, Peixoto FB, Marroquim OMG. Biosafity in dentistry: literature review. Braz J Health Review 3.2 (2020): 1656-62.

11. Farias ERR, Silva AMCS, Sousa INA Rocha KMBT, Leal JBP, Sousa VA. A percepção do usuário quanto a humanização no atendimento em unidades básicas de saúde do município de Floriano-Pi. CIAIQ. 2019;2:1497-501.

12. Neves M, Giordani JMA, Ferla AA, Hugo FN. Primary care dentistry in Brazil: from prevention to comprehensive care. J Amb Care Manag. 2017; 40(Sup.2):35-48.

13. Graff VA, Toassi, RFC. Clínica em saúde bucal como espaço de produção de diálogo, vínculo e subjetividades entre usuários e cirurgiões-dentistas da Atenção Primária à Saúde. Physis. 2018; 28(3):280-313.

14. Coelho IV, Melo ARF, Caetano RM, Silva CLM, Habibe RCH. Avaliação da satisfação do paciente atendido na Clínica Integrada Odontológica do UniFOA. Braz J Health Review. 2020; 3(1):673-83.
15. Bordin D, Fadel CB, Moimaz SAS, Garbin CAS, Saliba NE. Considerações de profissionais e usuários sobre o serviço público odontológico: um aporte para o planejamento em saúde. Rev APS. 2016; 19(2):221-9.

16. Oliveira RP, Monteiro PRS, Ribeiro AJM, Reis AC, Dias AC. Avaliação do atendimento ambulatorial do hospital universitário Clementino Fraga Filho. South Am Devel Soc J. 2020; 6(16):321-5.

17. Santos LP, Duarte MR, Caetano PL. Avaliação dos usuários sobre a qualidade do serviço prestado por graduandos do curso de odontologia do centro universitário Estácio Juiz de Fora: pesquisa clínica. Pey Këyo. 2020; 5(3):68-84.

18. Pêgo SPB, Dias VO, Nascimento JE, Junior HM, Martelli DRB, Santos ML, et al. Avaliação dos serviços odontológicos prestados por acadêmicos: percepção do usuário. Intercâmbio. 2016; 7:138-47.

\section{Correspondência para:}

Paulo Leonardo Ponte Marques

e-mail: paulomarques@unifor.br

Rua Andrade Furtado, 955/802 Cocó

60192-072 Fortaleza/CE 